\title{
Corela
}

Cognition, représentation, langage

HS-18 | 2015

La reformulation : usages et contextes

\section{Reformuler oralement le lexique des textes littéraires}

De la catégorisation du lexique à expliquer vers la modalisation du discours de l'enseignant

Julie Rançon

\section{OpenEdition}

Journals

Édition électronique

URL : http://journals.openedition.org/corela/4057

DOI : $10.4000 /$ corela.4057

ISSN : 1638-573X

Éditeur

Cercle linguistique du Centre et de l'Ouest - CerLICO

Édition imprimée

Date de publication : 15 novembre 2015

ISSN : 1638-5748

Référence électronique

Julie Rançon, «Reformuler oralement le lexique des textes littéraires », Corela [En ligne], HS-18| 2015,

mis en ligne le 16 novembre 2015, consulté le 30 avril 2019. URL : http://journals.openedition.org/

corela/4057 ; DOI : 10.4000/corela.4057

Ce document a été généré automatiquement le 30 avril 2019

\section{cc) (i) (2)}

Corela - cognition, représentation, langage est mis à disposition selon les termes de la licence Creative Commons Attribution - Pas d'Utilisation Commerciale - Partage dans les Mêmes Conditions 4.0 International. 


\title{
Reformuler oralement le lexique des textes littéraires
}

\author{
De la catégorisation du lexique à expliquer vers la modalisation du \\ discours de l'enseignant
}

Julie Rançon

1 La présente contribution porte sur les reformulations lors de l'étude de textes littéraires en classe de langue (Français Langue Maternelle ou Français Langue Etrangère et Seconde), reformulations considérées comme des éléments inhérents et constitutifs de l'interaction didactique (Volteau, 2009). De telles séquences de focalisation ont pour objectif de mettre en relief des éléments non connus par les apprenants et/ou plus ou moins difficiles à reformuler. Il semble en effet plus aisé de reformuler et de comprendre le lexème "arbre " plutôt que "l'immatériel », pour reprendre l'exemple de Bogaards (1994). Cette perspective définit le critère de difficulté de l'apprentissage du lexique par le degré de concrétude/d'abstraction d'une lexie ou d'une expression lexicale (au sens de Pottier, 1992) et se caractérise par le fait qu'une lexie est plus ou moins représentable par une image concrète, définie et structurée. Mais qu'en est-il dans le discours reformulatif de l'enseignant lors d'une interaction en classe?

2 Dans une étude récente, l'enseignant témoigne d'une activité cognitive spécifique en interaction et ce, qu'il ait préparé son cours en amont de la classe ou non (Rançon, 2009). Il peut monopoliser la parole ou partager la construction du sens entre tous les acteurs pédagogiques. C'est ce que Gülich et Kotschi nomment en 1987 l'auto-reformulation en opposition à l'hétéro-reformulation. Cette terminologie montre comment les enseignants problématisent une pensée, valident ou non des informations, tendent vers une compréhension effective du discours en somme, comment les enseignants diversifient individuellement leurs pratiques interactionnelles. Cette affirmation prend davantage de sens quand on la croise avec la proposition de Bogaards (1994) de penser la catégorisation lexicale comme représentative d'une échelle de difficulté pour reformuler.

3 Cette double perspective est orientée par une finalité didactique: analyser le fonctionnement et le contenu des interactions langagières au regard de la catégorisation du lexique à reformuler et ce, dans deux types de classes différentes (la classe de français 
langue maternelle et la classe de français langue étrangère ou seconde) qui ont pour objectif similaire de faire comprendre du lexique en français. Ainsi, compte tenu de ces paramètres multiples, nous pouvons émettre l'hypothèse que des marques discursives spécifiques vont émailler les discours des enseignants et ce, en corrélation avec les caractéristiques sémantiques des lexies à reformuler.

\section{Reformuler du lexique en classe de langue}

\subsection{La situation d'interaction}

Les séquences reformulatives en classe de langue peuvent prendre des formes langagières diversifiées, orales ou écrites (manuels scolaires, exposés, débats, explications de texte, par exemple). Notre propos concernera uniquement les reformulations fournies par l'enseignant à l'oral lors d'explications de texte littéraire parce que c'est moins l'activité globale que le format d'interaction localement accompli qui détermine le type de ressources mises en œuvre (Mondada \& Pekarek Doehler, 2001, 2004). Notons que les textes littéraires contiennent des mots, termes ou expressions difficiles à comprendre pour un apprenant qu'il soit de français langue maternelle ou de langue étrangère ou seconde. Ils sont définis par l'enseignant qui préparera ou non une explication en amont du cours. Si nous considérons le discours explicatif comme étant d'une part, l'explanandum (ce qui est à expliquer) et d'autre part, l'explanans (ce qui explique) (Hudelot, 2001), ce dernier peut être assimilé à une sorte de reformulation dont le sens serait, d'une part, explicatif, d'un autre, répétitif. Double problématique pour une structure qui réitère un signifié n'utilisant pas le même signifiant (Rey-Debove in ChesnyKohler, 1981 : 97). Le but étant de « faire comprendre » (De Gaulmyn, 1986), de répondre à une question explicite ou implicite restée sans réponse de la part des apprenants. A un niveau purement interactionnel, la participation interlocutive et la gestion de la classe peuvent être des facteurs de variation du discours reformulatif : soit ce discours reformulatif est monopolisé par l'enseignant, soit il est partagé dans la classe, soit il est dans un premier temps partagé puis glisse progressivement vers un monologue professoral.

Dans le premier cas, l'enseignant peut imposer sa vision et soumettre une reformulation préalablement préparée ou non aux apprenants. Il estime qu'en fonction des connaissances antérieures des élèves, le discours reformulatif fourni suffira à faire comprendre. L'enseignant est en position d'autorité (au sens de Bourdieu) et son discours fait loi. C'est une explication monologuée (Colletta \& Pellench, $2005: 6$ ) mais nous lui préfèrerons le terme d'auto-reformulation (Gülich \& Kotschi, 1987 ; De Gaulmyn, 1991 ; Rançon \& Spanghero-Gaillard, 2005 ; 2007) dans le sens où le discours explicatif est en soi un discours de reformulation et que l'enseignant prend à sa charge ce discours en vue de faire comprendre.

Dans le deuxième cas, l'enseignant incite ses apprenants à découvrir ensemble le sens du mot ou de l'expression. Il fait appel aux connaissances antérieures de chacun et laisse le discours se partager au sein de la classe. Cette co-construction du sens n'est possible que si les apprenants ont les connaissances suffisantes pour déduire/induire le sens du mot. C'est à l'enseignant qu'appartient le choix de proposer cette technique reformulative. Il a la nécessité de connaitre suffisamment bien ses apprenants (leurs connaissances, leurs représentations, leurs stratégies d'apprentissage, etc.) pour présenter un discours dont il n'aura pas la maitrise absolue. Il devra jouer le rôle de chef d'orchestre pour amener 
progressivement à la bonne compréhension de l'élément textuel. C'est une explication dialoguée (Colletta \& Pellench, $2005:$ 7), partagée entre les locuteurs. L'enseignant pourra paraphraser le discours des apprenants, il procèdera à une hétéro-reformulation (Gülich \& Kotschi, 1987 ; De Gaulmyn, 1991 ; Rançon \& Spanghero-Gaillard, 2005 ; 2007).

Dans le dernier cas, l'enseignant peut laisser le discours reformulatif se partager entre les membres de la classe mais va progressivement récupérer ce discours pour l'achever. Ce glissement discursif de l'hétéro-reformulation à l'auto-reformulation (Rançon, Spanghero-Gaillard, Dat \& Billières, 2008) opéré par l'enseignant peut être conscient ou inconscient. Ainsi, l'interaction première entre apprenants et enseignant s'éclipse progressivement en un monologue professoral. C'est lui qui aura le dernier mot sur l'identification de l'élément à découvrir alors que l'inverse sera quasi impossible. L'apprenant ne peut pas s'exprimer après un monologue de l'enseignant, en tout cas, cela semble peu probable lorsque les apprenants sont des novices (enfants, jeunes adultes ou adultes débutants).

6 Ces remarques mettent en exergue à quel point les acteurs de l'interaction sont les moteurs du discours reformulatif en interaction (cf. Pochon-Berger dans ce volume. Ils créent les conditions nécessaires à la production et à la compréhension du lexique. L'enseignant-vecteur, de son côté, tente d'organiser et de réguler les échanges. Il agit avec ses mots, ses manières, ses connaissances du monde. Son discours est alors empreint d'un lexique qui lui correspond, de structures syntaxiques qui l'identifient; d'une approche reformulative qui est sienne.

\subsection{Reformuler selon ses propres formules}

7 Le discours de l'enseignant contient des éléments reconnaissables par les apprenants comme étant sien. Il semble difficile d'observer deux discours reformulatifs identiques de deux enseignants de deux classes différentes, à l'exception d'avoir préparé en amont les reformulations en s'aidant d'un dictionnaire commun et en proposant telle quelle une définition. Cette reformulation définitoire correspond au degré maximum d'objectivité. C'est ce que Greimas (1976) appelle le degré zéro de modalisation.

8 Mais lors de l'interaction, l'enseignant laisse parler sa personnalité. Il explique en reformulant certains de ses propres syntagmes qui pourraient être marqués par des modalisations axiologiques, c'est-à-dire des marques discursives qui expriment l'affectivité du locuteur. Elles aident implicitement les apprenants à adhérer au discours. Elles dévoilent une argumentation spécifique propre à chaque enseignant.

9 A une autre extrémité, lors d'une hétéro-reformulation (la reformulation du propos des apprenants), l'enseignant interroge les apprenants et construit avec eux une définition lexicale. Ce discours pourrait être marqué par des modalisations axiologiques mais aussi par des modalisations positives ou négatives, c'est-à-dire des marques de l'appréciation que l'enseignant porte sur les propositions reformulatives des apprenants. Il s'agit d'un guidage discursif qui permet de valider ou d'infirmer les propositions des apprenants. Se joue alors une co-construction du sens qui peut mener à une étroite négociation des énoncés. Nous pouvons parler de cheminement hypothético-déductif (Py, 2005) plus ou moins contrôlé par l'enseignant et qui s'appuie sur les connaissances antérieures des apprenants. Ainsi, l'apprenant a participé activement à la construction des savoirs, ce qui en facilite sa compréhension, sa mémorisation dans la mémoire à long terme et sa réutilisation potentielle dans une autre situation de communication (Kail \& Fayol, 2003). 

de reformuler. Que ce soit en Français Langue Maternelle (désormais FLM) ou en Français langue Etrangère et Seconde (FLES), les enseignants choisissent personnellement leur(s) façon(s) de présenter un discours reformulatif en fonction de la situation d'enseignement-apprentissage. C'est un choix individuel qui ne semble pas provenir d'une appartenance quelconque à une méthodologie précise. Ils peuvent monologuer leurs reformulations ou les dialoguer en fonction du contexte de communication (Rançon, 2009).

11 Ces considérations permettent de penser que les discours reformulatifs sont riches de construction, de co-construction du sens mais le lexique à reformuler n'en est-il pas l'essence même? N'est-il pas à l'origine de cette interaction didactique?

\section{Le type de lexique à reformuler}

12 Nous utiliserons le terme de lexie (Pottier, 1987/1992) pour désigner les éléments non connus par l'apprenant et susceptibles d'être reformulés par l'enseignant. «La lexie est le groupe de morphèmes intégrés qui constitue l'unité de signification. C'est une unité fonctionnelle, vraisemblablement mémorisée en compétence» (Rastier, Cavazza \& Abeillé, 1994: 47). Le terme de lexie correspond à ce qui peut être expliqué et de fait, reformulé dans un texte. Il peut s'agir d'une lexie comprenant un seul morphème comme la préposition « à » ou bien composée de plusieurs morphèmes. Les lexies sont des unités de sens (Pottier, 1992) qui font partie soit de la compréhension globale, soit de la compréhension détaillée du texte littéraire. Ces lexies peuvent être simples ou complexes. Pottier (idem) parle d'unité lexicale (UL) pour une lexie simple, c'est-à-dire que le sens est perceptible par le lecteur au premier abord. Au contraire, une lexie complexe associe un sens supplémentaire qu'il n'est pas forcément aisé de reconnaitre. On peut inclure dans cette catégorie les métaphores, métonymies, synecdoques mais aussi les lexies nécessitant des opérations cognitives interprétatives en fonction du contexte, du cotexte, etc. Ainsi l'usage décontextualisé d'une lexie ne suffit pas à notre propos, l'enseignant se sert de l'usage contextualisé de la lexie en situation d'enseignement. Il peut s'agir d'une activation, inhibition ou propagation de sèmes et qui transforment les significations répertoriées en langue (Rastier, Cavazza \& Abeillé, 1994: 69). Dans notre expérimentation, ont été choisies des lexies simples et complexes, représentatives des lexies contenues dans les textes littéraires tels que Bel-ami de Maupassant. Ont été également pris en considération les discours explicatifs contextualisés afin de définir les différentes opérations cognitives mises en œuvre par les enseignants. En effet, ces derniers procèdent en faisant des hypothèses sur ce que les apprenants sont susceptibles de savoir. L'enseignant établit ainsi une échelle de valeur de degré de difficulté aux items lexicaux. Bogaards (1994) parle plus précisément du degré de concrétude qui établit une échelle de valeur entre ce qui peut être facile/difficile pour un apprenant.

\subsection{Le degré de concrétude/d'abstraction des lexies}

Ce critère de difficulté de l'apprentissage se caractérise par le fait qu'une lexie est plus ou moins représentable par une image comprise par l'apprenant. Weis (1986: 178, in Bogaards, 1994: 152) montre que le problème de concrétude ne s'arrête pas à l'apprentissage d'items nominaux. Il explique premièrement que les lexies abstraites sont 
plus difficiles à comprendre que les lexies concrètes et insiste sur le fait que les verbes, les adverbes et les adjectifs sont plus difficilement retenus que les noms abstraits. Il prétend que ces classes de lexies seraient "moins rebelles à l'illustration visuelle», or lorsque l'on consulte un dictionnaire imagé, seuls des substantifs renvoyant à des objets concrets sont présents. Cependant, nous pouvons dire que les actions représentées en langue française par des verbes sont «figurables » par le mime, ou l'image animée. Il en va de même pour certains adverbes. Par contre, les adjectifs sont souvent dépendants d'un jugement de valeur de celui qui s'exprime à propos de quelque chose ou quelqu'un.

Plaut \& Shallice (1993) montrent également dans leurs recherches que les lexies concrètes comporteraient plus de traits sémantiques que les lexies abstraites. Par exemple, «cheval », lexie concrète renvoie à un référent dont les caractéristiques visibles sont la taille, le nombre de pattes, le pelage, la couleur, etc. La relation avec le domaine du visible, du palpable est mise en exergue dans les travaux de Paivio $(1986,1991)$ notamment avec sa théorie du double-encodage. La lexie bénéficierait d'un encodage verbal mais aussi d'un encodage non verbal (qui relèverait du perceptuel) alors que les lexies abstraites ne comprendraient qu'un encodage verbal expliquant de fait leur plus grande « volatilité » en mémoire.

Il a été ainsi observé que les lexies concrètes sont plus faciles à apprendre, à définir, à comprendre et à réutiliser que les concepts abstraits. De même, les lexies concrètes sont les premières à être apprises par les enfants. Et lors d'une tâche d'écriture ou de lecture, les lexies concrètes sont plus facilement lues et écrites par les apprenants en L1. Les travaux psycholinguistiques révèlent également que l'apprenant a un empan plus grand pour les lexies concrètes que pour les lexies abstraites, illustrant ainsi une meilleure récupération des lexies concrètes que des lexies abstraites en mémoire de travail (Baddeley, 1992 : 121-122).

\subsection{Hypothèses de recherche}

Suite à ces travaux, nous faisons l'hypothèse que reformuler une lexie concrète est plus facile que reformuler une lexie abstraite. En effet, les discours reformulatifs de lexies concrètes par l'enseignant bénéficieraient de références perceptuelles (images, sons, description physique, imitation de gestes, etc.) qui pourraient être utilisées en classe lors de l'explication.

17 Par ailleurs, nous fondant sur notre propre expérience d'enseignante de FLE, il nous est apparu que plus la lexie à expliquer appartient au domaine du concret (renvoie à une réalité) plus elle est facile à définir et plus l'enseignant partage son discours reformulatif avec les apprenants. Le phénomène d'hétéro-reformulation accompagnerait alors le discours explicatif des lexies concrètes.

En revanche, l'enseignant qui doit expliquer une lexie qui relève du sensible, domaine plus abstrait, aurait davantage besoin de partir de ses propres connaissances et de reformuler ses propos (et donc d'auto-reformuler). Il devrait alors s'impliquer dans le discours de manière à faire adhérer son auditoire et userait de marqueurs modalisateurs (verbaux, paraverbaux et/ou non-verbaux).

19 Ainsi s'impose dans notre travail une analyse sémique (Rastier, 2009) des différentes lexies et propositions lexicales contenues dans les textes littéraires. Cette analyse permettrait de mettre en évidence le contenu de la lexie à partir de la décomposition du 
sens en unités minimales. Une telle démarche définit ainsi si une lexie contient des sèmes concrets ou abstraits. Elle offre la perspective d'en déduire que la lexie appartient davantage à une catégorie (concrète) plutôt qu'une autre (abstraite).

\section{Les données de notre expérimentation}

Ceci nous conduit, sur le plan épistémologique, à inscrire notre étude dans une approche didactique des processus interactionnels (Mondada \& Pekarek Doelher, 2000) fondée sur les travaux en cognition située. De fait, les données de notre expérimentation tentent de respecter le déroulement d'une séquence de cours sur la compréhension orale de texte. En observant et filmant sept classes de langue, nous avons pu comparer les mouvements interactionnels inhérents à la reformulation lexicale. La vidéo a également permis d'identifier les éléments verbaux, paraverbaux (prosodie dont l'intonation, le débit, le rythme, les pauses) et non-verbaux (gestes, mimiques et utilisation de supports iconiques) utilisés par les acteurs pédagogiques. Tout ce corpus (soit 27h de film) a été transcrit à partir des conventions de transcription ICOR de l'Equipe ICAR (Université Lyon II Lumière).

\subsection{Les enseignantes de FLM et de FLES}

21 Plus précisément, nous avons proposé à cinq enseignants de FLM (deux professeurs de collège, $3^{\text {ème }}$ et trois professeurs de lycée, $2^{\text {nde }}$ ) et deux enseignants de FLE (en Université) de travailler sur trois textes littéraires, trois textes de Bel-Ami de Maupassant. Les enseignants choisis pour participer à notre expérimentation ont été sélectionnés sur deux critères essentiels : les professeurs sont tous d'origine française et ils ont suffisamment d'ancienneté ou de formation dans leur métier pour être en mesure d'avoir un discours sur leurs pratiques, de sorte que ces enseignants nous apparaissent comme étant représentatifs du personnel d'enseignement employé dans et hors fonction publique du Ministère de l'Education Nationale et du Ministère de l'Enseignement Supérieur et de la Recherche. Ces hommes et femmes ont des cursus scolaires et universitaires spécifiques, des expériences propres et des manières singulières de concevoir l'enseignementapprentissage du français. Cependant, les enseignants de FLM et de FLES n'ont pas les mêmes publics, et les objectifs pédagogiques (linguistiques, communicatifs, culturels, etc.) diffèrent en fonction des instructions officielles (pour les FLM) et des recommandations des équipes pédagogiques universitaires (pour les FLES). Pourtant, l'objectif commun reste l'enseignement de connaissances sur le français en langue française.

Leurs représentations personnelles sur l'acquisition de connaissances par les apprenants se retrouvent également dans leurs discours pédagogiques et dans les stratégies d'enseignement mises en place par chacun des acteurs et précisent leurs objectifs à atteindre. Nous nous attendons à observer de multiples techniques explicatives, tant les personnalités et les parcours universitaire et professionnel de chacun divergent (cf. annexe). 


\subsection{Les approches méthodologiques des enseignants de FLM et de FLES}

mieux appréhender les représentations des enseignants sur leurs approches méthodologiques, nous leur avons proposé un questionnaire à remplir où il était question de définir ce qu'était un enseignement pour eux, qu'entendaient-ils par " apprentissage " et comment concevaient-ils l'enseignement-apprentissage du lexique à la suite de la compréhension de textes littéraires (environ une dizaine de questions sur le sujet). Pour les enseignants (tous confondus), " enseigner " ne signifie pas seulement proposer des textes d'auteur même si c'est ce qu'ils font en classe. Cette proposition leur semble restrictive et ne représente pas ce qu'est un enseignement du lexique. Ceci dit, proposer des textes d'auteurs est inscrit dans les textes officiels en FLM et l'est aussi dans la trame proposée aux enseignants de FLES pour la troisième année d'apprentissage du français à l'université de Toulouse. Au collège, les enseignants doivent pouvoir présenter des textes illustrant la grande richesse de la langue française et faire acquérir aux élèves une culture littéraire commune. Ils les amènent, avec l'appui de toutes les disciplines enseignées, à s'exprimer clairement, à l'écrit comme à l'oral (Bulletin Officiel, 2004). "Au lycée, l'approche de l'histoire littéraire et culturelle se fait de façon plus réflexive. Elle permet de saisir les grandes scansions historiques que constituent les changements majeurs dans les façons de penser et de sentir, mais aussi dans les façons de s'exprimer. (...) En fonction des difficultés de lecture que présentent les cuvres relevant d'un état de langue historiquement éloigné, l'attention portera davantage, sans exclusive cependant, sur des textes et mouvements littéraires des XIXème et XXème siècles en seconde, et sur des textes et mouvements littéraires et culturels antérieurs en première " (Bulletin Officiel, 2001). Les textes d'auteurs proposés en classe nécessitent d'être nombreux et variés. A l'université de Toulouse, au département d'étude de français langue étrangère, les enseignantes observées ont pour mission de faire découvrir aux étudiants un large panel de l'histoire littéraire française. Au travers de textes d'auteurs et au fil des siècles, les enseignantes présentent un panorama rapide de l'évolution de la littérature et des idées en France. L'étude de trois textes littéraires, à savoir trois passages de Bel-Ami de Maupassant, est ainsi légitimée dans toutes les classes observées.

Il est à noter également que les enseignants ont choisi eux-mêmes la manière avec laquelle ils présenteraient et étudieraient les textes; ils ont eu une réelle liberté pédagogique. Aucune condition pédagogique ne leur a été imposée (ni condition de temps, ni condition matérielle, etc.). Les enseignants de $2^{\text {nde }}$ ont alors longuement travaillé sur les textes (6 heures en moyenne) alors que les enseignants de $3^{\text {ème }}$ les ont étudiés rapidement ( $1 \mathrm{~h}$ par texte), ce qui se justifie pleinement, étant donné que les objectifs à atteindre ne sont pas les mêmes. Les enseignants de FLE, n'étant pas soumis à un curriculum prédéfini, ont personnellement choisi de traiter les textes de manière à ce qu'ils s'intègrent au mieux au programme semestriel (l'un a consacré $2 \mathrm{~h}$ à l'étude des trois textes, l'autre 4 heures).

En outre, à la suite des réponses obtenues lors de notre questionnaire écrit, nous avons pu définir les approches méthodologiques des enseignants de notre expérimentation. Nous résumerons les résultats obtenus en notifiant que les enseignants adoptent en majorité une conception (plutôt) socioconstructiviste de l'apprentissage (construction de connaissances à partir des connaissances antérieures, d'une part) et qu'ils estiment également opter pour une vision assez cognitiviste de l'enseignement (création de savoir- 
faire, d'autre part). Ainsi, les représentations qu'ils ont sur leur propre enseignement n'est certainement pas sans conséquence lors de l'apprentissage de connaissances par les apprenants.

\subsection{Les apprenants de FLM et de FLES}

Ces derniers sont des adolescents et jeunes adultes (français et étrangers, toutes nationalités) qui vivent en France. Les élèves français sont en $3^{\text {ème }}$ de collège (14-15 ans) et $2^{\text {nde }}$ de lycée général (15-16 ans), comme indiqué précédemment.

Les étudiants étrangers proviennent de tous pays, sont de niveau B2 selon le CECR et ont été évalués en tant que tel par leurs professeurs. Cela signifie qu'ils ont une maitrise efficace et spontanée de la langue mais qu'ils sont à un moment de leur apprentissage où l'objectif final est le suivant (Conseil de l'Europe, $2001: 25)$ :

B2 : Peut comprendre le contenu essentiel de sujets concrets ou abstraits dans un texte complexe, y compris une discussion technique dans sa spécialité. Peut communiquer avec un degré de spontanéité et d'aisance tel qu'une conversation avec un locuteur natif ne comportant de tension ni pour l'un ni pour l'autre. Peut s'exprimer de façon claire et détaillée sur une grande gamme de sujets, émettre un avis sur un sujet d'actualité et exposer les avantages et les inconvénients de différentes possibilités.

Certains apprenants ont acquis ces compétences et tendent pour la plupart vers un niveau C1. D'autres sont encore en difficulté face à des sujets abstraits contenus dans un texte complexe. Le CECR insiste sur cette donnée de concret/abstrait qui prend toute sa mesure entre le niveau B1 et le niveau B2 (Conseil de l'Europe, 2001 : 82, 89, 115, 123, 125, 166). Cette donnée est prépondérante dans notre étude dans le sens où l'enseignant a potentiellement conscience de ces difficultés et tentera de reformuler ses propos ou ceux des apprenants en tenant compte de ce paramètre.

\subsection{Correspondances linguistiques des publics de FLM et FLES}

Nous ne pouvons pas établir de correspondance directe entre le niveau des apprenants FLM et celui des apprenants FLES. Vigner ${ }^{1}$, ancien inspecteur académique, connaissant parfaitement les disciplines du FLM et du FLES, estime que les performances attendues de la part des apprenants de FLM ne font pas l'objet d'une définition organisée comme peut le proposer le CECR pour les publics de FLES. L'enseignement du FLM est un composé de compétences (lire, écrire, parler, écouter) dans des domaines et usages du discours (textes littéraires plus particulièrement et textes documentaires) et de contenus (culture littéraire et grammaire).

L'école fonctionne sur la base d'un consensus fondé sur une estimation purement empirique. Vigner se demande d'ailleurs s'il n'est pas plus utile de fonctionner de la sorte au vue du nombre d'enseignants ( 60000 professeurs de français pour le secondaire) et du nombre d'apprenants (5 millions d'élèves). Il ajoute ensuite que le brevet des collèges et le baccalauréat s'attachent à évaluer les compétences et performances des apprenants mais qu'ils ne peuvent être rattachés aux niveaux du CECR. Encore que, il a tenté, avec un collègue du lycée français de Barcelone, d'étalonner les épreuves écrites du brevet des collèges au CECR. Cet outil, qui ne peut être pris comme solution absolue, montre toute la volonté d'établir un référentiel de compétences commun aux langues étrangères et aux langues maternelles. Les résultats situent l'examen du brevet des collèges, à peu près, au 
niveau C1. Les apprenants de FLES ont été évalués par nos soins au niveau B2 (tendant vers un niveau $\mathrm{C} 1$ dans ce module de littérature). Ils peuvent ainsi être jugés comme étant quasi-comparables.

31 En outre, les apprenants de FLM et de FLES ont développé des compétences scolaires et universitaires identiques en compréhension (orale et écrite) de textes littéraires. Les apprenants de FLES sont plus âgés et ont une meilleure connaissance du monde. Leurs savoirs encyclopédiques pallient les difficultés de langage. En cela, nous pensons pouvoir comparer assez facilement les différents publics.

Par ailleurs, les enseignantes de FLES (LEC et GOU) ont accepté de collaborer à notre étude et de travailler sur les trois textes proposés, à savoir Bel-Ami de Maupassant. Elles ont jugé individuellement que leurs apprenants pouvaient comprendre ces textes. Les enseignants de lycée (GAJ, MIC et LAZ) ont participé à l'étude de ces textes parce qu'ils intégraient les recommandations pédagogiques préconisées par le Ministère de l'Education Nationale : le roman d'apprentissage doit être vu en classe de seconde. Les enseignants de collège (BRE et PLA) ont également accepté d'expliquer Bel-Ami parce qu'il permettait de "revenir sur des notions vues en début d'année: le récit, les différents types de discours, la narration, la description, le portrait, les paroles ou pensées rapportées, les figures de style " (PLA, réponse au questionnaire destiné aux enseignants). BRE atteste de la même façon que l'étude de ces trois textes permet «la confirmation de certaines connaissances». Il ajoute à la réponse de PLA que ces textes admettent d'étudier «le roman réaliste, la description et ses procédés: temps verbaux, expansion du groupe nominal, registres, connotations, point de vue, etc.» (BRE, réponse au questionnaire destiné aux enseignants).

\subsection{Le lexique des textes littéraires}

Les enseignants n'en oublient pas que le lexique qui compose ces textes mérite d'être explicité afin d'en dégager le sens textuel. A ce sujet, le lexique difficile à comprendre et nécessaire à reformuler provient de trois passages de Bel-Ami de Maupassant. Ces textes comprennent des lexies simples et complexes, peu usitées dans la langue courante ou vieillies. 31 lexies et propositions lexicales ont été considérées par les enseignants comme difficiles à comprendre et potentiellement nécessaires à reformuler. Le terme de « difficile » peut être assimilé à « nécessaire à expliquer, à expliciter, à éclaircir » selon les enseignants. Il a été aussi considéré comme non compris par des élèves de $2^{\text {nde }}$. Ces derniers, ne faisant pas partie de notre expérimentation, ont eu pour tâche de souligner tous les mots et expressions qu'ils ne comprenaient pas dans les textes. Nos résultats montrent qu'il existe une correspondance entre les lexies soulignées par les élèves et celles choisies par les enseignants. Cependant, un certain nombre de lexies ne peut pas forcément être mis en relief par les apprenants. Les lexies dont le sémantisme est double ou métaphorique ne sont peut-être pas apparues aux yeux des apprenants («boite noire » pour désigner «le fiacre", "rôdeuses " pour « des prostituées»). Tout l'art revient à l'enseignant qui saura faire la lumière sur des termes qui semblaient à première vue anodins mais qui cachent d'autres sens. C'est principalement pour cette raison que nous ne pouvons pas nous contenter de nos lycéens-informateurs pour établir une liste lexicale posant des difficultés de compréhension. Nous avons également soumis nos lexies au critère de fréquence de la base de données BRULEX. Ces dernières ont été considérées comme étant peu fréquente dans la langue. Cependant, il n'a pas été possible de soumettre nos propositions lexicales telles que « il lui semblait qu'il allait faire un bond 
du portique de la Madeleine au portique du Palais Bourbon » à cette base de données. Ces propositions lexicales sont si spécifiques de par leur signification contextuelle et cotextuelle qu'elles peuvent également être considérées comme non usitées dans la langue courante.

Ainsi, les lexies et propositions lexicales retenues comptent parmi elles: «des polissonneries », « un hussard », « coudoyer », " une divinité », « impérieux », « de boni », "des bocks", " une élégance tapageuse ", etc. Seulement, les enseignants n'ont pas forcément choisi les mêmes lexies et/ou ne les ont pas forcément expliquées, même si elles avaient été préparées en amont du cours. Notre regard s'est alors porté sur les éléments qui avaient été reformulés par le plus grand nombre d'enseignants et pour lesquels nous avons mené l'analyse sémique (Rastier, Cavazza \& Abeille, 1994) suivante :

\begin{tabular}{|c|c|c|}
\hline \multicolumn{3}{|c|}{ Constituant n'ayant que des traits sémantiques concrets } \\
\hline Lexie simple & $\begin{array}{l}\text { Une gargote à } \\
\text { prix fixe }\end{array}$ & $\begin{array}{l}\text { /lieu/+/manger/+/qualité -/+/cher -/ } \\
\text { /prix/+/variable -/ }\end{array}$ \\
\hline $\begin{array}{l}\text { Lexie } \\
\text { complexe }\end{array}$ & Une boite noire & $\begin{array}{l}\text { Usage :/appareil/+/avion/+/enregistrement/+/données/ } \\
\text { Connotation :/moyen de locomotion/+/époque/+/fiacre/ } \\
\text { Dénotation, caractéristiques sémantiques :/couleur sombre/ } \\
\text { +/forme carrée/ }\end{array}$ \\
\hline Lexie simple & Une étuve & / lieu/+/+chaleur/+/provoque la sudation/ \\
\hline \multicolumn{3}{|c|}{ Constituant ayant une majorité de traits sémantiques abstraits } \\
\hline $\begin{array}{l}\text { Lexie } \\
\text { complexe }\end{array}$ & Bel-Ami & $\begin{array}{l}\text { Hors contexte : / humain/+/beauté +/+/amitié +/ } \\
\text { En contexte :/humain/+/homme/+/personnage principal/ }\end{array}$ \\
\hline Lexie simple & Des miasmes & $\begin{array}{l}\text { Sens } 1: \\
\text { /émanations/+/matières/+/décomposition/+/bactéries/ + / } \\
\text { maladies+/ } \\
\text { Sens } 2: \\
\text { /sensation/+/odorat/+/négatif/+/matières/+ } \\
\text { décomposition/ }\end{array}$ \\
\hline Lexie simple & $\begin{array}{l}\text { Une élégance } \\
\text { tapageuse }\end{array}$ & $\begin{array}{l}\text { Elégance (hors contexte) :/acte humain/+/esthétique+/+/ } \\
\text { qualité/+/goût+/+/habillement/+/personne/ } \\
\text { Tapageuse (hors contexte) : } \\
\text { /acte humain/+/ouïe/+/négatif/+/bruit/ } \\
\text { Tapageuse en contexte associé à élégance : } \\
\text { /acte humain/+/voyant/+/qualité/+/goût -/ }\end{array}$ \\
\hline
\end{tabular}

L'analyse sémique a pour objectif principal de définir les sèmes généraux et spécifiques qui composent les constituants des textes de Bel-Ami. Si les traits de la lexie contiennent des sèmes relevant de la sensation ou de l'immatériel, ils seront qualifiés de "sémantiquement plutôt abstraits" en opposition à des lexies "sémantiquement 
concrètes ». Cette étude permet de définir plus clairement et objectivement ce qui peut relever du difficile ou du facile d'une part à comprendre et à mémoriser pour un apprenant et d'autre part à reformuler de la part de l'enseignant.

Ainsi, les lexies simples « une gargote à prix fixe » et " une étuve » ne contiennent que des traits sémantiques concrets. /Provoquer la sudation/ contient un trait sémantique plutôt abstrait si l'on considère l'action « provoquer » mais pouvant être imagé ou mimé de part le degré de concrétude initié par "la sudation». Cette expression nous parait alors moins abstraite que la représentation matérielle de "l'amitié » présente dans la lexie «Bel-Ami ». Il devrait être alors moins difficile pour l'apprenant de reconstituer le sens de chaque constituant que pour des lexies ayant une majorité de traits sémantiques abstraits. Enfin, les lexies simples "des miasmes» et "une élégance tapageuse » contiennent une majorité de traits sémantiques abstraits. Ce sont ces lexies qui devraient poser principalement des soucis de compréhension et de construction de sens pour les apprenants.

\section{L'analyse des résultats obtenus}

Les résultats qualitatifs obtenus suite à l'analyse de notre corpus ont montré que la notion de trait sémantique abstrait semble être en relation avec les marques de modalisation (verbales, paraverbales et non-verbales du discours reformulatif, plus présentes lorsque la lexie est auto-reformulée. Nous pouvons dire que l'enseignant qui explique un mot qui relève du sensible, domaine plus abstrait, a davantage besoin de partir de ses propres connaissances et de reformuler ses propos. Il s'implique alors dans le discours de manière à faire adhérer son auditoire et use de marqueurs modalisateurs axiologiques.

Voici un extrait d'auto-reformulation dans le corpus reformulatif de l'enseignante LEC (FLES) dans lequel nous remarquons qu'il y a beaucoup de modalisations axiologiques: « des miasmes », lexie comportant des traits sémantiques plutôt abstraits.

Extrait de l'enseignante LEC (FLES) lors de l'explication « des miasmes »

LEC et alors un miasme (2.8) là on reprend l'idée de haleines empestaient des
égouts (2.4) alors miasme $c^{\prime}$ est un vocabulaire assez spécifique $d^{\prime}$ accord $(0.2)$
$c^{\prime}$ est une émanation hein donc émanation $c^{\prime}$ est quelque chose qui se dégage (0.3)
euh une (émas $;$ imana $;$ émanation) pardon assez dangereuse (0.5) et qui se
dégage de de $(0.3)$ de matière en décomposition (0.6) hein en train de pourrir
quoi (0.2) si vous voulez (.) hein $c^{\prime}$ est $c^{\prime}$ est quand même du vocabulaire (.)
évidemment comme $c^{\prime}$ est une émanation euh donc qui part euh qui se dégage de
corps en décomposition euh de corps de produits de matière etcetera (0.3)
évidemment l'odeur qui se dégage de de $(($ petits rires)) (0.5)〉 est très
désagréable (0.5) hein (.) d'accord (0.3) tout ça c'est euh (.) il faut
imaginer évidemment de revenir au paris du dix-neuvième siècle euh (0.3) bon
(0.2) évidemment on avait pas le même système d'égout qu'on avait aujourd'hui
(1)

Dans cet extrait de corpus, au niveau qualitatif, nous remarquons que nous sommes en présence de modalisations paraverbales et non-verbales. Elles se déclinent sous différentes formes : la prosodie, l'utilisation de supports iconiques mais aussi tout ce qui relève de la posturo-mimo-gestualité et de la proxémique. Dans notre cas, l'enseignante LEC utilise principalement la prosodie. L'enseignante use également de modalisations 
verbales dans son discours reformulatif en vue de se positionner face au dire (hésitations, prudence dans les propos), d'avoir un comportement verbal modérateur (nuancer ou insister sur des propos), d'introduire une certaine évaluation (positive ou négative) ou de modaliser pour demander des informations, des explications, de l'attention.

Contrairement à cela, les discours reformulatifs de lexies aux traits sémantiques concrets reposent sur des références perceptuelles (images, sons, description physique) qui peuvent être utilisées en classe lors de l'explication et qui n'imposent pas à l'enseignant de s'impliquer personnellement dans le discours. L'enseignant peut impliquer les apprenants dans le discours et hétéro-reformuler. Les discours de l'enseignant sont jalonnés de marqueurs modalisateurs positifs ou négatifs. Les discours reformulatifs semblent s'échafauder en interaction en relation avec les propriétés intrinsèques des lexies à expliquer.

41 Dans notre corpus, l'hétéro-reformulation appelle davantage de modalisations positive et/ou négative que de modalisations axiologiques lors de la reformulation d'une lexie aux traits sémantiques majoritairement concrets. L'exemple suivant est assez représentatif des discours observés :

Extrait de l'enseignante GOU (FLES) lors de l'explication de « une boite noire »

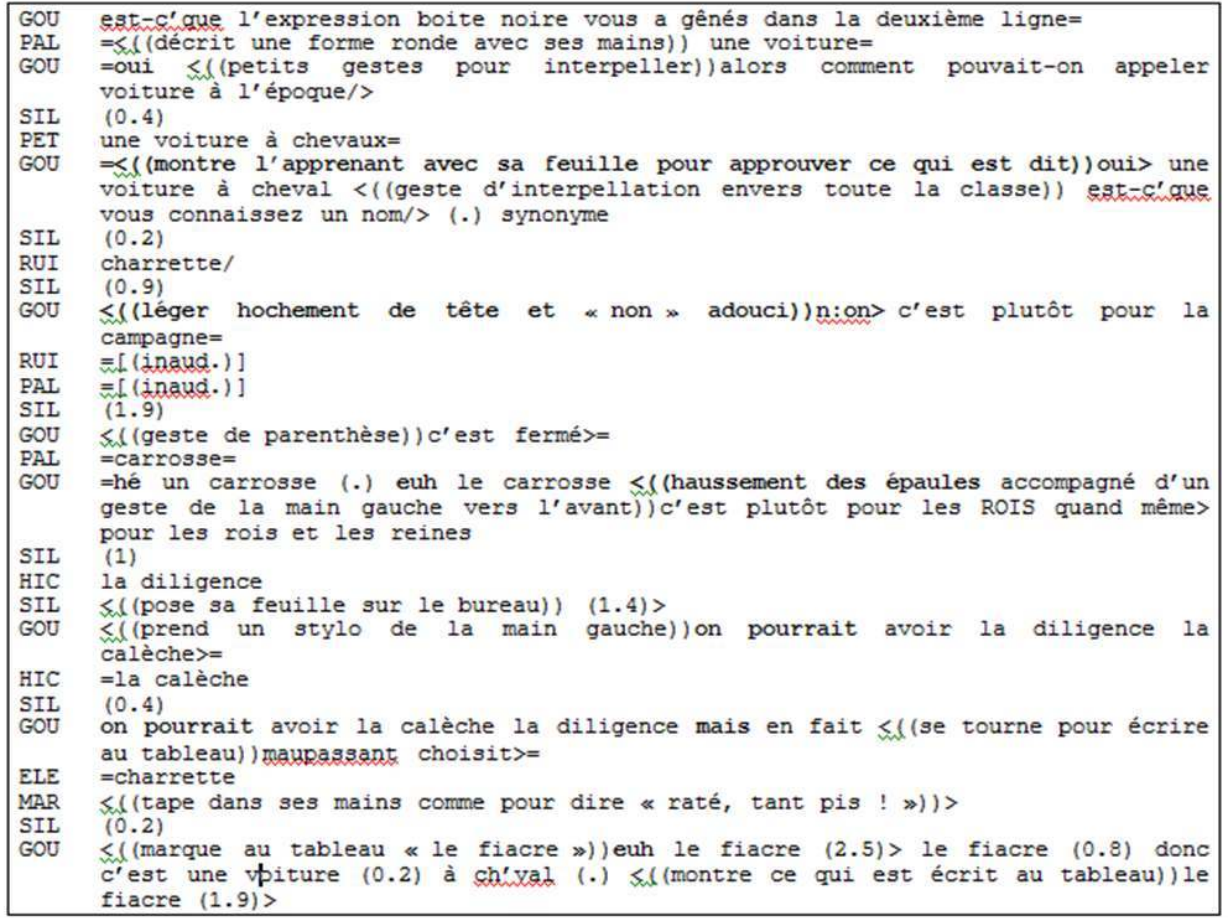

42 GOU guide les apprenants à la recherche du sens exact de la lexie et parsème son discours d'évaluations. Ainsi, les hétéro-reformulations contiennent davantage de modalisations positives ou négatives qui ont pour objectif de guider les apprenants vers la bonne compréhension de la lexie.

43 Enfin, lors de glissements discursifs de l'hétéro-reformulation à l'auto-reformulation observés dans notre corpus, le type de modalisations se modifie au cours du discours reformulatif en interaction d'une lexie contenant des traits sémantiques majoritairement abstraits. Il passe d'une modalisation positive ou négative à une modalisation axiologique, comme dans l'exemple suivant :

Extrait de l'enseignant BRE (FLM) lors de l'explication de « une élégance tapageuse » 


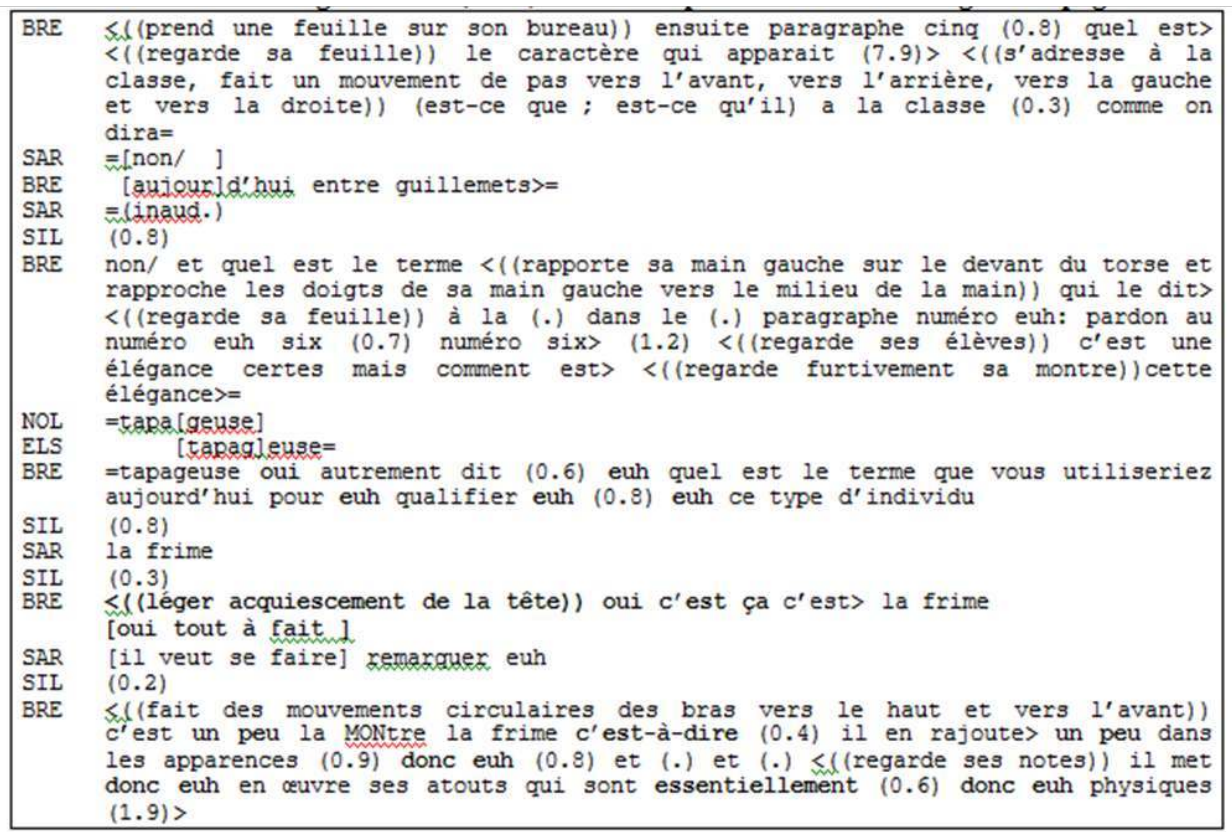

$44 \mathrm{Au}$ moment où BRE auto-reformule, il semble avoir besoin de justifier son propos et intègre à son discours des modalisations axiologiques qui étaient absentes auparavant. Le début du discours reformulatif était émaillé de modalisations positives ou négatives et ne laissait transparaitre aucune implication personnelle de l'enseignant. En revanche, l'autoreformulation implique que les locuteurs transforment leur manière de présenter le discours reformulatif en interaction.

Au niveau quantitatif ${ }^{2}$, à présent, il est important de noter qu'il n'existe pas de différence reformulative significative entre les enseignants de FLM et de FLES. Ils obtiennent quantitativement les mêmes résultats et opèrent tous de la même manière. En observant le comportement discursif de tous les enseignants lors des explications orales des quatre lexies les plus expliquées (deux aux traits sémantiques plutôt abstraits, deux aux traits sémantiques concrets), l'attitude reformulative diverge non pas en fonction de qui ils sont (FLES ou FLM) mais en fonction de la lexie qui est à expliquer. Concernant les deux lexies concrètes, "étuve" et "gargote ", les enseignants ont laissé les apprenants prendre en charge la reformulation. Ils ont hétéro-reformulé. Quantitativement, le nombre de modalisations est inférieur à 14 pour la lexie «étuve » et 21 pour la lexie "gargote». A l'opposé, l'enseignant semble modaliser beaucoup moins que dans une auto-reformulation. En effet, lorsqu'il reformule une lexie abstraite, l'enseignant a tendance à auto-reformuler et ses propres propos contiennent beaucoup de marqueurs modalisants (en bleu). Nous pouvons comptabiliser 35 modalisations pour la lexie « miasmes » et 117 modalisations pour la lexie « Bel-Ami ».

Constituants aux traits sémantiques concrets ou majoritairement concrets 

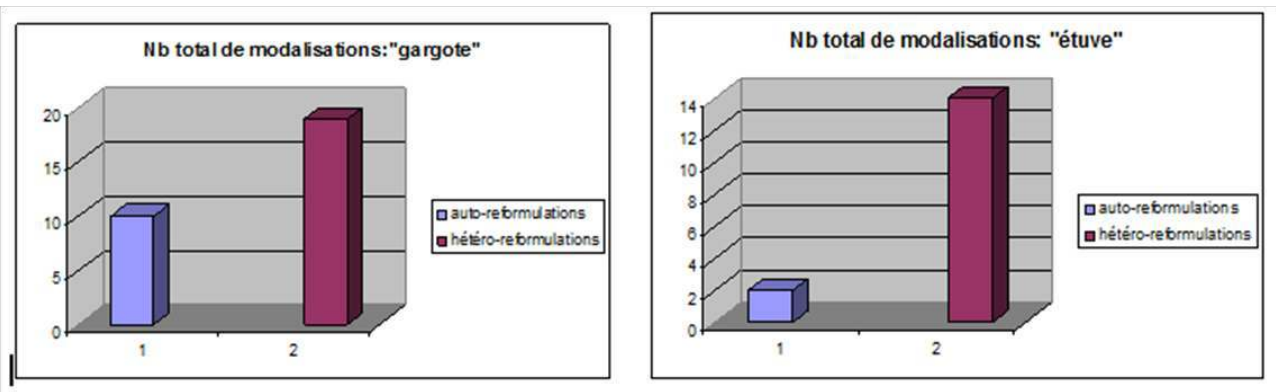

Constituants aux traits sémantiques abstraits ou majoritairement abstraits :
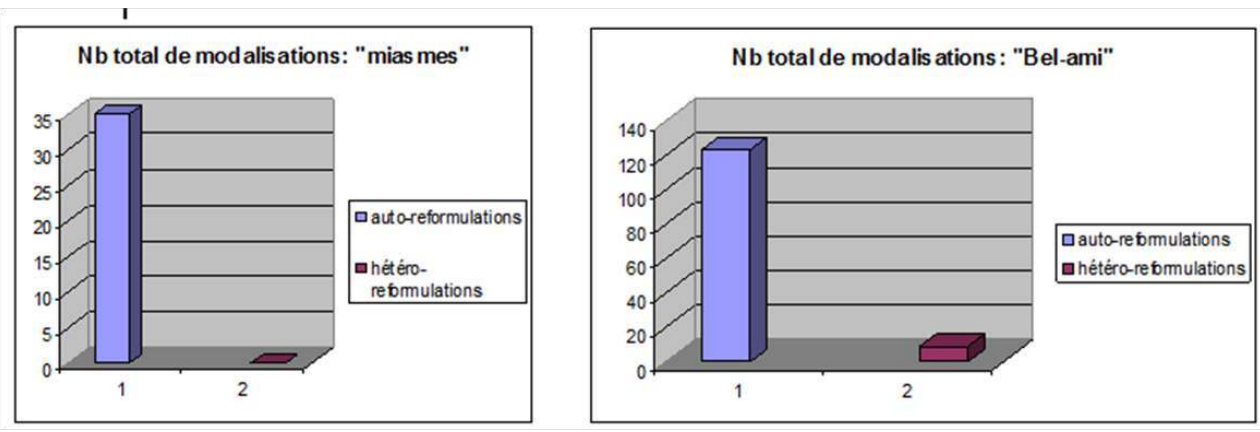

Il en est de même dans l'analyse d'une autre lexie sémantiquement abstraite " il portait beau» (Lexie synthétique: /humain/+/comportement $+/+$ /prestance $+/$ ) où trois enseignants (MIC (FLM), GAJ (FLM) et GOU (FLES)) l'ont expliquée à l'aide d'autoreformulations et ont utilisé un nombre conséquent de modalisations axiologiques. Le quatrième enseignant (LEC, enseignante de FLES) l'a expliquée en opérant un glissement de l'hétéro-reformulation vers l'auto-reformulation et le temps passé à auto-reformuler est supérieur à celui consacré à hétéro-reformuler. Nous observons aussi dans ce cas un grand nombre de modalisations axiologiques.

Pour les lexies dont les traits sémantiques sont concrets, le phénomène inverse est moins marqué. Deux enseignants (PLA (FLM) et GOU (FLES)) ont expliqué la lexie sémantiquement concrète " hussard » à l'aide d'hétéro-reformulations qui sont marquées par de nombreuses modalisations positives et/ou négatives. Seule l'enseignante LEC (FLES) a opéré un glissement discursif de l'hétéro-reformulation vers l'autoreformulation qui contient de nombreuses marques axiologiques (sachant que LEC a passé plus de temps à auto-reformuler qu'à hétéro-reformuler).

Enfin, les résultats obtenus pour la lexie sémantiquement concrète « une gargote à prix fixe » confirment tout ce qui a été dit précédemment. Lorsque l'enseignant autoreformule, il intègre à son discours de nombreuses marques axiologiques alors qu'en hétéro-reformulant, les modalisants sont soit positifs, soit négatifs. Et lorsqu'un glissement discursif s'observe, il est fortement assimilé au fonctionnement d'une autoreformulation dans le sens où le temps consacré à auto-reformuler est plus important que celui consacré à hétéro-reformuler ; la modalisation axiologique prend numériquement le dessus.

Nous pouvons en conclure, au regard de la classification sémantique abstrait/concret, que le discours reformulatif de l'enseignant se modélise assez bien, qu'il soit enseignant de FLM ou enseignant de FLES. Reste à noter que quantitativement, les reformulations des lexies dont les traits sémantiques sont abstraits sont très souvent assurées par les 
enseignants et modalisées axiologiquement. A l'inverse, les lexies aux traits sémantiques concrets sont plutôt laissées à expliquer par la classe (et appuyées par des modalisations positives ou négatives). Les glissements discursifs observés d'hétéro-reformulation vers l'auto-reformulation, assez nombreux dans notre corpus, montrent que, quelle que soit la catégorisation sémantique de la lexie, cette dernière est principalement appuyée par une modalisation axiologique.

Fonctionnement discursif lors d'explications de lexies comportant des traits sémantiques abstraits

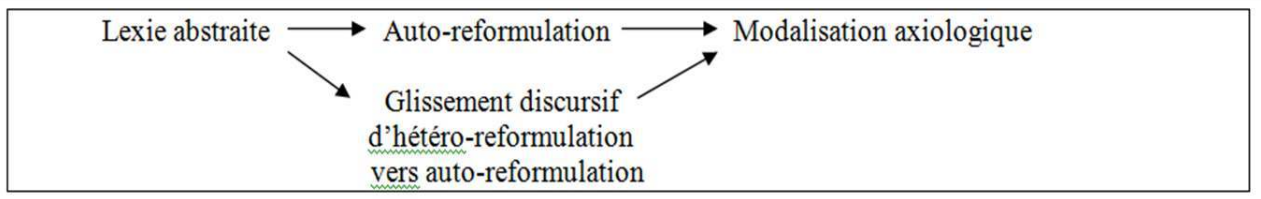

Fonctionnement discursif lors d'explications de lexies comportant des traits sémantiques concrets

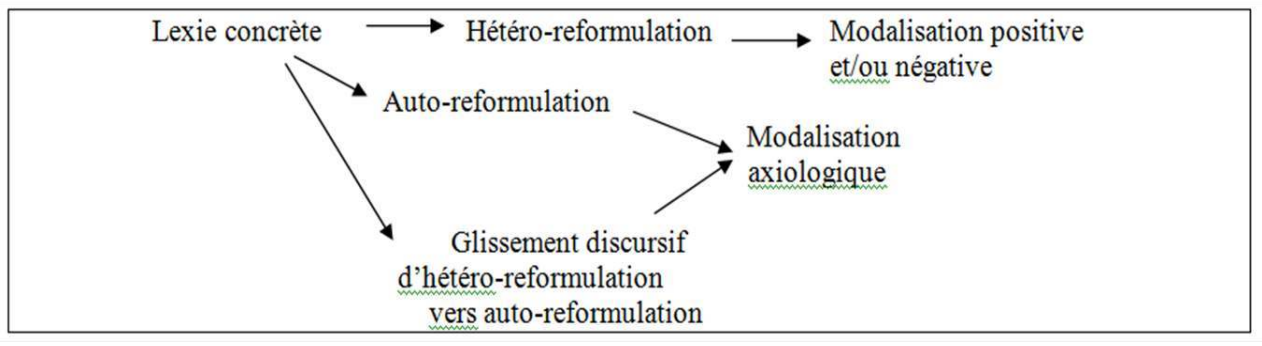

Il faut quand même souligner qu'au niveau interindividuel certains enseignants modalisent plus que d'autres. Ce sont les cas de LEC (enseignante de FLES) et GAJ (enseignante de FLM). Cependant, malgré les différences quantitatives des marques de modalisations, le constat est le même. Tous les enseignants ont tendance à autoreformuler en utilisant beaucoup de marqueurs modalisants axiologiques lorsqu'ils expliquent une lexie dite sémantiquement abstraite et à modaliser positivement et/ou négativement dans une hétéro-reformulation lorsqu'ils expliquent une lexie dite sémantiquement concrète.

\section{Discussion en guise de conclusion}

51 Nous rappelons que notre étude s'inscrit dans une double perspective: identifier les marques modalisatrices contenues dans une reformulation interactionnelle tout en prenant en considérant la catégorisation du lexique à expliquer. Les résultats obtenus amènent à plusieurs commentaires et réflexions que nous souhaiterions à présent évoquer.

Tout d'abord, la notion de lexie abstraite n'est pas si facile à appréhender. La distinction marquée entre les termes abstraits et concrets manque tout de même de pertinence objective. On peut se demander pour qui une lexie est abstraite et pour qui elle ne l'est pas. Les lexies utilisées dans notre recherche n'étaient pas connues des apprenants mais cette question pourrait se poser pour des experts d'un domaine. Baumard (2003) prend l'exemple des termes utilisés en géométrie et met ainsi en évidence que ces items renvoient à des objets mathématiques caractérisables par les experts du domaine alors qu'ils sont abscons pour un non-expert. Le degré d'expertise et donc des connaissances spécifiques portant sur un domaine, doit être associé au critère de concrétude. Ainsi, il renvoie à un critère dit de familiarité. Nous proposons alors d'envisager la concrétude 
d'un terme sur une échelle (plus ou moins abstrait/concret) pour telle ou telle personne caractérisée par ses connaissances du monde.

Par ailleurs, Flaux \& alii (1996), nous invitent à considérer deux dimensions : le mot dans la langue et le mot dans la parole (en contexte). Les mots abstraits sont dépendants linguistiquement et ontologiquement : ils ont besoin d'un sujet du point de vue linguistique (cas des verbes et adjectifs) et du point de vue de la réalité (référent). S'ils ont un référent, il semblerait qu'ils aient un double-encodage. Ainsi, la distinction abstrait/ concret ne tient plus seulement à la distinction matériel/ immatériel, qui pose de nombreux problèmes terminologiques, mais plus exactement à une question relevant de la référentialité autonome/non autonome du nom. Les noms ayant des occurrences «matérielles» comme référent sont considérés comme autonomes. Nommés catégorématiques, ils s'opposent à syncatégorématiques, c'est-à-dire des noms dont le référent n'a pas d'existence autonome. En comparaison à «fiacre» dont le référent « voiture » est autonome, «rôdeuse » ne l'est pas, parce que son référent « rôde » n'a pas «la trinité ontologique matière-forme-temps » (Galmiche \& Kleiber, 1996 : 35) lui permettant d'être indépendant. Ainsi, il est plus difficile pour un apprenant (étranger ou non) de saisir le sens d'un nom syncatégorématique car il ne peut pas le raccrocher à un référent «matériel». Cela ne veut pas dire qu'il connait le référent mais que l'association sémantique sera plus facile à établir entre les termes lors d'une explication orale ou écrite.

54 En résumé, nous pensons que l'opposition abstrait/concret est aussi fondée sur notre expérience du monde et l'ontologie qui lui est sous-jacente (Rançon \& SpangheroGaillard, 2007). Une personne ayant de grandes connaissances en équitation n'aura aucune difficulté à mettre une signification sur «un chambon" (un enrênement spécifique) ou " un hackamore » (une embouchure sans mors). En revanche, un non-initié ne pourra pas en découvrir seul le sens. Notre corpus est composé de lexies difficiles qui ne font pas partie des connaissances antérieures des apprenants. Donc, dans une acception large, les lexies sélectionnées peuvent être considérées comme étant toutes abstraites, c'est-à-dire au sens d'inaccessibles. Et même si le référent semble autonome, le contexte peut rendre inaccessible le sens du mot. La «fourchette » d'un cheval n'est pas un instrument culinaire mais la partie inférieure du sabot. Dans notre corpus, les lexies comme «femme du monde » posent des problèmes de compréhension car même si les lexies «femme» et «monde» sont référentiellement autonomes et familières, l'association des termes appelle une autre signification.

Par conséquent, en regardant de plus près le fonctionnement intrinsèque des lexies, nous pouvons dégager des traits sémantiques concrets et des traits sémantiques abstraits des lexies qui apparaitront pour l'apprenant plus ou moins concrètes/ plus ou moins abstraites. Martin (1996: 49-50) parle de procédure d'abstraction ou de procédure de concrétisation par rapport aux traits sémantiques des lexies. La tentative de typologie présentée est fort intéressante mais peut facilement intégrer des contre-exemples. Pour exemple unique, Martin (1996) parle d'abstraction hypéronymique catégorisant les hypéronymes comme étant plus abstraits que les hyponymes. Ainsi «animal» est plus abstrait que « cheval » dont les traits sémantiques plus nombreux sont considérés comme concrets.

Enfin, le discours reformulatif d'une lexie en interaction dépend du degré de connaissance de l'enseignant (qu'il soit de FLM ou de FLES, degré issu de connaissances ou d'un vécu. Un enseignant qui aura une grande connaissance en peinture pourra rendre 
concret des concepts abstraits parce qu'il saura à quoi la lexie fait référence. Il usera de moins de modalisations pour la faire comprendre à son auditoire. En ce sens, les marques de modalisation seraient plutôt des indices du degré de réalité que recouvrent les lexies pour l'enseignant.

Alors que traditionnellement les notions de concret/abstrait relèvent de mesures générales (cf. la psycho-physique), il semblerait plus à propos de les aborder individuellement et de les relativiser. Il nous apparait intéressant de partir de la catégorisation implicite des enseignants analysable par le biais des marques de modalisation (Dubois, 1991) et de parler à ce moment-là de plutôt concret/plutôt abstrait, +/- concret/abstrait selon les individus. A partir de ces catégories, nous pourrions trouver les corrélations qui existent pour l'analyse de la lexie. Il ne faut pas oublier cependant que les marques de modalisation sont des indices parmi d'autres de la catégorisation des lexies.

Ainsi, dans une formation de futurs enseignants de langue (en Master FLE ou à l'ESPE), nous pouvons intégrer ces remarques en vue de sensibiliser l'enseignant à la manière dont il s'exprime et dont il use du discours reformulatif en classe. L'enseignant, qui fait une discrimination personnelle de ce qui est à reformuler ou non, a la nécessité de s'interroger sur le degré d'abstraction et de concrétude de la lexie. Il comprendra que la préparation en amont de son discours reformulatif témoigne de la réalité que recouvrent les lexies selon son prisme personnel.

\section{BIBLIOGRAPHIE}

BAUMARD Nicolas, 2003, "Qu'est-ce qu'un mot abstrait ? », DEA de sciences cognitives, EHESS, [En ligne], dernière consultation le 18 juin 2008, disponible sur http://lumiere.ens.fr/ $\sim$ cogmaster/www/doc/MEMOIRES/2003_BAUMARD.pdf

BOGAARDS Paul, 1994, "Le vocabulaire dans l'apprentissage des langues étrangères », Hatier, Coll. Langues et apprentissage des langues, Paris, 255p.

BADDELEY Alan, 1992, «Working memory », Coll. Oxford psychology series, Clarendon press, Oxford, 289p.

COLLETTA Jean-Marc \& PELLENCH Catherine, 2005, « Les coverbaux de l'explication chez l'enfant âgé de 3 à 11 ans ", Interacting bodies, [En ligne], consulté le 27 janvier 2009, disponible sur http:// gesture-lyon2005.ens-lsh.fr/article.php3?id_article=223

CONSEIL DE L'EUROPE, 2001, «Cadre européen de référence pour les langues », Didier, Paris, 191p.

DE GAUMLYN Marie-Madeleine, 1991, « Expliquer des explications », Dausendschön-Gay \& Kraft (Eds.), Linguistische Arbeiten, Linguistische Interaktionsanalysen, Max Niemeyer Verlag, Tübingen, 279-314.

DUBOIS Danièle (dir.), 1991, « Sémantique et cognition. Catégories, prototypes, typicalité », Editions du CNRS, Coll. Sciences du langage Paris, 342p. 
FLAUX Nelly, GLATIGNY Michel \& SAMAIN Didier (Eds.), 1996, « Les noms abstraits : histoire et théories ", Actes du colloque de Dunkerque, Presses universitaires du Septentrion, Villeneuved'Ascq, 406p.

GALMICHE Michel \& KLEIBER Georges, 1996, « Sur les noms abstraits », "Les noms abstraits. Histoire et théories ", Flaux, Glatigny et Samain (Eds.), Presses universitaires du Septentrion, Villeneuve d'Ascq, 23-40.

GREIMAS Algirdas Julien, 1976, « Pour une théorie des modalités », Le langage, N³, Larousse, Paris.

GÜLICH Elisabeth \& KOTSCHI Thomas, 1987, « Les actes de reformulation dans la consultation «La dame de Caluire » in Bange (Ed.), «L'analyse des interactions verbales. La dame de Caluire », Peter Lang, Berne, 15-81.

KAIL Michèle \& FAYOL Michel (dir.), 2003, «Les sciences cognitives et l'école », PUF, Paris, 497p.

MARTIN Robert, 1996, " Le fantôme du nom abstrait ", " Les noms abstraits. Histoire et théories », in Flaux, Glatigny \& Samain (Eds.), Presses universitaires du Septentrion, Paris, 41-50. MONDADA Lorenza \& PEKAREK DOELHER Simona, 2001, « Interactions acquisitionnelles en contexte. Perspectives théoriques et enjeux didactiques ", Le français dans le monde (Recherches et application) , numéro spécial, 107-142.

MONDADA Lorenza \& PEKAREK DOELHER Simona, 2004, « Second Language Acquisition as Situated Practice : Task Accomplishment in the French Second Language Classroom ", The Modern Language Journal, $\mathrm{N}^{\circ} 88(4), 501-518$.

PAIVIO Allan, 1986, « Mental Representations: a dual coding approach », Oxford University Press, New-York, 322p.

PLAUT David C. \& SHALLICE Tim, 1993, « Deep dyslexia : A case study of connectionist neuropsychology », Cognitive Neuropsychology, 10, 377-500.

POTTIER Bernard, 1987/1992, " Théorie et analyse en linguistique », Hachette Supérieur, Coll. Langue Linguistique Communication, Paris, 240p.

PY Bernard, 2005, « La construction interactive de la norme comme pratique et comme représentation ", Acquisition et Interaction en Langue Etrangère, $\mathrm{N}^{\circ} 12$, [En ligne], Approches interactionnistes de l'acquisition des langues étrangères, dernière consultation le 22 juin 2007, disponible sur : http://aile.revues.org/document1464.html

RASTIER François, CAVAZZA Marc \& ABEILLE Anne, 1994, « Sémantique pour l'analyse. De la linguistique à l'informatique », Masson, Coll. sciences cognitives, Paris, 240p.

RANÇON Julie \& SPANGHERO-GAILLARD Nathalie, 2005, « La reformulation dans l'explication. En quoi les études de français langue étrangère aident-elles à comprendre ce processus ?», communication affichée, Colloque national « Répétitions, Reformulations, Reprises. Quels usages dans les interactions verbales?", organisé par le groupe Pergame, $1^{\mathrm{er}}-2$ avril2005, Université René Descartes, Paris.

RANÇON Julie \& SPANGHERO-GAILLARD Nathalie, 2007, « Place et rôle de la modalisation dans le discours explicatif en classe de langue ", Journées franco-suédoises de linguistique, "Les modalisations du discours ", communication orale, du 7-9 juin 2007, Uppsala.

RANÇON Julie, SPANGHERO-GAILLARD Nathalie, DAT Marie-Ange \& BILLIERES Michel, 2008, «Impact du discours explicatif de l'enseignant en classe de FLE : Réflexions sur la formation des enseignants ", Actes du XIIème Congrès Mondial de la FIPF (Fédération Internationale de Professeurs de 
Français) «Faire vivre les identités francophones dans le monde », du 20 au 25 Juillet 2008, Québec

(Canada), (soumis). www.fipf-quebec2008.com

RASTIER François, 2009, Sémantique interprétative, PUF, Paris.

VOLTEAU Stéphanie, 2009, « Les reformulations orales dans les interactions didactiques au cycle 3 de l'école primaire. Formes et fonctions des reformulations d'une enseignante expérimentée et d'une enseignante débutante dans une classe de CM2 », thèse de doctorat, Université de Toulouse II Le Mirail, Toulouse, 277p.

\section{ANNEXES}

\section{Annexes}

Description précise des enseignants :

Le groupe de participants-enseignants de FLM se compose de deux enseignants de collège (niveau $3^{\text {ème }}$ ) et trois enseignants de lycée d'enseignement général ( $\left.2^{\text {nde }}\right)$. Leurs parcours universitaire et professionnel sont résumés dans le tableau ci-dessous :

\begin{tabular}{|c|c|c|c|c|c|}
\hline $\begin{array}{ll}\text { Nom } & \mathrm{de} \\
\text { l'enseignant } & \end{array}$ & PLA & BRE & MIC & GAJ & LAZ \\
\hline Sexe & Homme & Homme & Femme & Femme & Homme \\
\hline Age approximatif & 55 ans & 54 ans & 38 ans & 26 ans & 45 ans \\
\hline $\begin{array}{l}\text { Institution } \\
\text { d'appartenance }\end{array}$ & $\begin{array}{l}\text { Collège } \\
\text { Vasconie, } \\
\text { Miélan }^{3} \\
\text { (Gers) } \\
\begin{array}{l}\text { Classe de } 3 \\
\text { ème }\end{array}\end{array}$ & $\begin{array}{l}\text { Collège } \\
\text { Vasconie, } \\
\text { Miélan } \\
\text { (Gers) } \\
\begin{array}{l}\text { Classe de } 3 \\
\text { ème }\end{array}\end{array}$ & $\begin{array}{l}\text { Lycée général } \\
\text { de Pardailhan, } \\
\text { Auch }{ }^{4} \text { (Gers) } \\
2^{\text {nde générale }}\end{array}$ & $\begin{array}{l}\text { Lycée général } \\
\text { de } \\
\text { Pardailhan, } \\
\text { Auch (Gers) } \\
2^{\text {nde générale }}\end{array}$ & $\begin{array}{l}\text { Lycée Saint } \\
\text { Sernin, } \\
\text { Toulouse } \\
\text { (Haute- } \\
\text { Garonne) } \\
\text { Classe de } 2^{\text {nde }} \\
\text { générale }\end{array}$ \\
\hline $\begin{array}{l}\text { Niveau } \\
\text { diplôme le plus } \\
\text { élevé }\end{array}$ & $\begin{array}{l}\text { Licence } \\
\text { Lettres } \\
\text { Modernes }\end{array}$ & $\begin{array}{l}\text { Maitrise } \\
\text { Lettres } \\
\text { Classiques }\end{array}$ & $\begin{array}{l}\text { Licence Lettres } \\
\text { Classiques }\end{array}$ & $\begin{array}{l}\text { Licence } \\
\text { Lettres } \\
\text { Modernes }\end{array}$ & $\begin{array}{l}\text { Licence } \\
\text { Lettres } \\
\text { Modernes }\end{array}$ \\
\hline Spécialisation & $\begin{array}{l}\text { Arts } \\
\text { plastiques }\end{array}$ & Latin/grec & Latin/grec & $\varnothing$ & $\begin{array}{lr}\text { Mention } & \text { FLE, } \\
\text { travail } & \text { sur } \\
\text { l'image } & \end{array}$ \\
\hline $\begin{array}{l}\text { Formation } \\
\text { pédagogique }\end{array}$ & $\begin{array}{l}\text { École } \\
\text { normale }\end{array}$ & $\begin{array}{l}\text { École } \\
\text { normale }\end{array}$ & $\begin{array}{l}\text { Sans } \\
\text { formation } \\
\text { pédagogique }\end{array}$ & IUFM & IUFM \\
\hline
\end{tabular}




\begin{tabular}{|l|l|l|l|l|l|}
\hline Statut d'emploi & $\begin{array}{l}\text { Enseignant } \\
\text { titulaire } \\
\text { second } \\
\text { degré }\end{array}$ & $\begin{array}{l}\text { Enseignant } \\
\text { titulaire } \\
\text { second } \\
\text { degré }\end{array}$ & $\begin{array}{l}\text { Maitre } \\
\text { auxiliaire, } \\
\text { enseignante } \\
\text { non-titulaire }\end{array}$ & $\begin{array}{l}\text { Enseignante } \\
\text { titulaire } \\
\text { second degré }\end{array}$ & $\begin{array}{l}\text { Enseignant } \\
\text { titulaire, } \\
\text { premier et } \\
\text { second degré }\end{array}$ \\
\hline $\begin{array}{l}\text { Années } \\
\text { d'enseignement } \\
\text { en FLM }\end{array}$ & 35 ans & 34 ans & 10 ans & 4 ans $s^{5}$ & 10 ans \\
\hline $\begin{array}{l}\text { Années } \\
\text { d'enseignement } \\
\text { au total }\end{array}$ & 35 ans & 34 ans & 14 ans & 4 ans & $\begin{array}{l}\text { ans } \\
\text { (instituteur } \\
\text { auparavant) }\end{array}$ \\
\hline $\begin{array}{l}\text { Ancienneté au } \\
\text { sein d'institutions } \\
\text { d'enseignement }\end{array}$ & Fin carrière & Fin carrière & Milieu carrière & $\begin{array}{l}\text { Début } \\
\text { carrière }\end{array}$ & $\begin{array}{l}\text { Milieu } \\
\text { carrière }\end{array}$ \\
\hline
\end{tabular}

Description des enseignants de FLM

Les enseignants peuvent être titulaires ou non de l'Education Nationale. Titulaires, ils ont reçu un enseignement pédagogique délivré par les Ecoles Normales (auparavant) ou par les ESPE (actuellement). Mais il est possible d'être enseignant du secondaire sans jamais avoir reçu de formation didactique. C'est le cas de MIC qui a commencé sa carrière en effectuant des remplacements et qui n'a jamais obtenu le CAPES interne ${ }^{7}$. Elle a acquis le titre de maitre auxiliaire et aurait dû, après un certain nombre d'années en tant que remplaçante, être titularisée.

Le programme de la formation pédagogique des enseignants ayant changé au fil des ans pour répondre aux besoins évolutifs des apprenants, nous estimons que l'enseignement pédagogique reçu par BRE et PLA n'aura pas la même incidence que chez GAJ ou LAZ. Leurs formations didactiques se référaient à des modèles d'enseignement-apprentissage différents de ceux faisant l'objet de formations plus récentes.

Nous supposons également que la formation à une spécialisation (arts plastiques, latin/ grec, FLE et travail de l'image) a des incidences sur la manière d'aborder une leçon de français. Les cours pourraient être émaillés de procédés explicatifs empruntés à d'autres disciplines (nous pensons notamment à la projection de scènes et tableaux comme cela est courant en arts plastiques).

Nous aurions souhaité une équité entre le nombre d'enseignants de FLM et celui de FLES. Mais cela n'a pas pu être possible pour des raisons administratives. En outre, les classes de niveau avancé ne sont pas très nombreuses et seuls l'université de Toulouse II-Le Mirail et l'Institut Catholique de Toulouse en comptent au moins une. Les autres institutions proposent des cours de FLES mais à des niveaux plus faibles et optent pour un enseignement communicationnel de la langue où des cours purement littéraires sont anecdotiques. Nous avons dû nous contenter de l'observation de deux enseignantes, que nous découvrons de la manière suivante :

\begin{tabular}{|l|l|l|}
\hline Nom de l'enseignant & GOU & LEC \\
\hline
\end{tabular}




\begin{tabular}{|l|l|l|}
\hline Sexe & Femme & Femme \\
\hline Age approximatif & 50 ans & 28 ans \\
\hline Institution d'appartenance & Université Toulouse II le Mirail & $\begin{array}{l}\text { Université Toulouse II } \\
\text { le Mirail }\end{array}$ \\
\hline Niveau du diplôme le plus élevé & DEA linguistique appliquée & Maitrise FLE \\
\hline $\begin{array}{l}\text { Cursus précédent } \\
\text { Formation pédagogique }\end{array}$ & Lettres Modernes, FLE & $\begin{array}{l}\text { Licence } \\
\text { Modernes mention FLE }\end{array}$ \\
\hline Années d'enseignement en FLE & 16 ans & Formation FLE \\
\hline $\begin{array}{l}\text { Années d'enseignement au } \\
\text { total }\end{array}$ & 30 ans & 5 ans \\
\hline Emploi précédent & Institutrice à l'étranger & Dilieu carrière en FLE, fin carrière \\
\hline Ancienneté & carrière en tant qu'enseignante & Dans \\
\hline
\end{tabular}

Description des enseignantes de FLES

Le cursus universitaire de ces enseignantes comprend une formation à l'enseignement du FLES. Et la particularité principale de ces professeurs de FLES à l'université est qu'ils occupent un emploi secondaire en tant que vacataire. GOU et LEC travaillent, toutes les deux, dans des écoles de langues privées où elles dispensent la majorité de leurs heures de cours. Dans le cas de GOU, qui était institutrice à l'étranger, cette dernière est revenue en France et a repris ces études pour obtenir un DEA de linguistique. Elle a perdu son statut de fonctionnaire en optant pour un emploi plus instable mais plus intéressant à son goût. On peut dire également que cette personne est en fin de carrière si l'on considère toutes ses années d'enseignement mais qu'elle est en milieu de carrière dans le domaine du FLES. Elle s'oppose à LEC qui est en début de carrière et qui se rapproche clairement du profil de GAJ. Nous pensons que les représentations qu'elles ont d'un enseignement de langue s'effectueront à partir des modèles qu'elles auront eus durant leur formation pédagogique.

Premier passage de Bel-Ami de Maupassant:

"Quand la caissière lui eut rendu la monnaie de sa pièce de cent sous, Georges Duroy sortit du restaurant (...) on voyait briller les gros cylindres transparents de glace qui refroidissaient la belle eau claire. » 


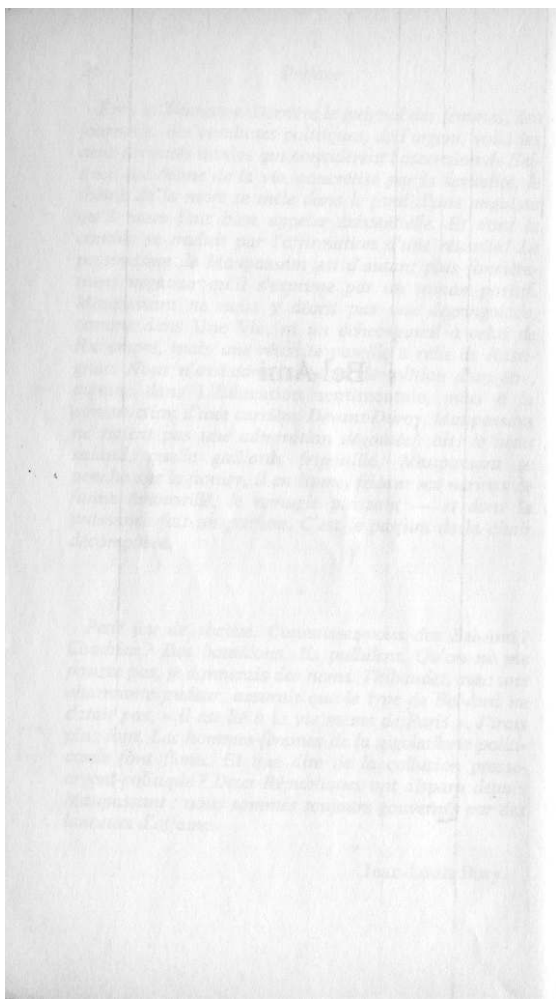

30

Bel-Ami

encore deux collations au pain et au saucisson, plus deux bocks sur le boulevard. C'était là sa grande dépense et son grand plaisir des nuits; et il se mit à descendre la rue Notre-Dame-de-Lorette. Il marchait ainsi qu'au temps où il portait l'uniforme des hussards, la poitrine bombée, les jambes un peu entrouvertes comme s'il venait de descendre de cheval; et il avançait brutalement dans la rue pleine de monde, heurtant les épaules, poussant les gens pour ne point se deranger de sa route. Il inclinait assez defrat to lair de toujours defier que pave de son talon. Il avait

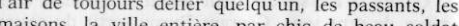
Quoique habillé dôn

Quoique habile dun complet de soixante francs, il gardait une certaine élégance tapageuse, un peu d'un blond châtain vaguement roussi, avec une moustache retroussée, qui semblait mousser sur sa lèvre, des yeux bleus, clairs, troués d'une pupille toute une raie au milieu du crâne, il ressemblait bien au mauvais sujet des romans populaires.

C'était une de ces soirées d'été où l'air manque dans Paris. La ville, chaude comme une étuve, paraissait suer dans la nuit étouffante. Les égouts soufflaient par leurs bouches de granit leurs haleines empestées, et les cuisines souterraines jetaient a la rue, par leurs fenetres basses, les miasmes infames des eaux de Les conces

des chises de pille, furce de chemise, à chur des chaises de paille, fumaient la pipe sous les portes front nu, le chapeau a la main.

Quand Georges Duroy parvint au boulevard, il petite, des cheveux frisés naturellement, séparés par
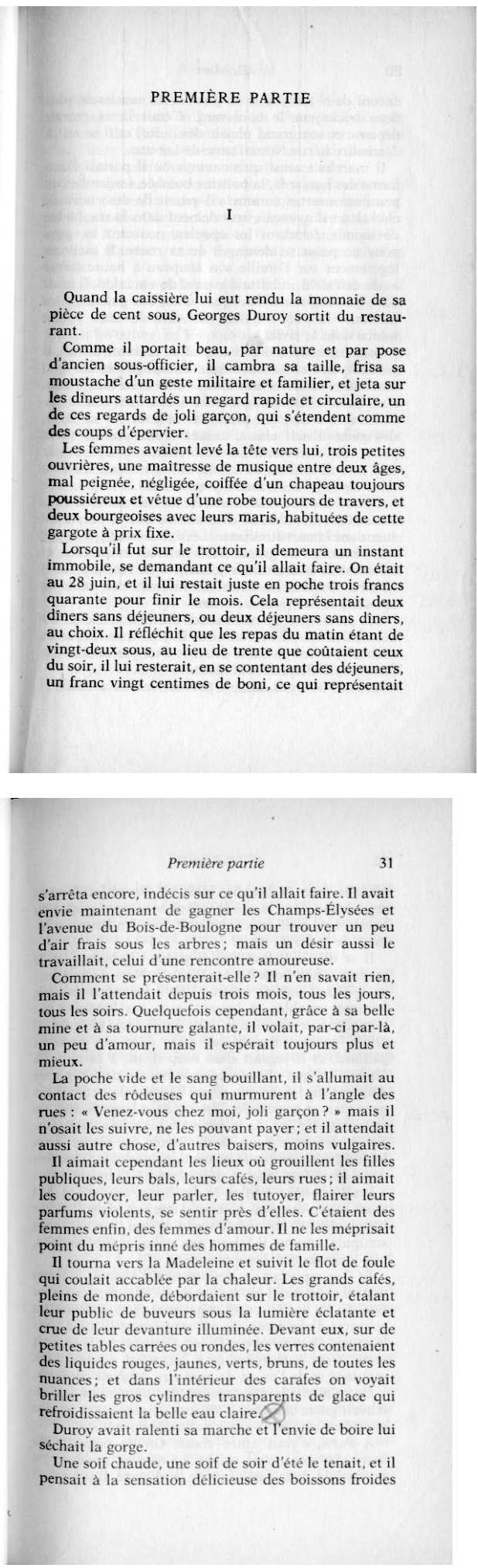

\section{Deuxième passage de Bel-Ami de Maupassant :}

«Il la sentait contre lui, si près, enfermée avec lui dans cette boite noire (...) une femme du monde ! du vrai monde ! du monde parisien! Comme ça avait été facile et inattendu !» 
préoccupés de ce qu'ils disaient, plongés dans un bain d'amour.

Les deux femmes, maintenant, en lançaient de roides, $\mathrm{M}^{\mathrm{me}}$ de Marelle avec une audace naturelle qu ressemblait à une provocation, $\mathrm{M}^{\mathrm{me}}$ Forestier avec un réserve charmante, une pudeur dans le ton, dans la voix, dans le sourire, dans toute l'allure, qui soulignait, en ayant l'air de les atténuer, les choses hardies sorties de sa bouche.

Forestier, tout à fait vautré sur les coussins, riait, buvait, mangeait sans cesse et jetait parfois une parole tellement osee ou tellement crue que les formes, un peu choquees par la forme et pour la trois, prenaient un peit air gene qui durait deux on nerie trop grose, il ajout : Vou a que polissonenfants. Si voss continuez comme ca, vous finin, mes faire des bêtises.

Le dessert vint, puis le café; et les liqueurs versèrent dans les esprits excités un trouble plus lowd tet phe chaud.

Comme elle l'avait annoncé en se mettant à table, avec une grâce étaie et bavarde ete femme qui accentue, pour amuser ses convives, une pointe d'ivresse très réelle.

$\mathrm{M}^{\mathrm{me}}$ Forestier se taisait maintenant, par prudence peut-être; et Duroy, se sentant trop allumé pour ne pas se compromettre, gardait une réserve habile.

On alluma des cigarettes, et Forestier, tout à coup, se mit à tousser.

Ce fut une quinte terrible qui lui déchirait la gorge; et, la face rouge, le front en sueur, il étouffait dans sa serviette. Lorsque la crise fut calmée, il grogna, d'un air furieux : - Ça ne me vaut rien, ces parties-là; c'es

stupide. Toute sa bonne humeur avait disparu dans la terreur du mal qui hantait sa pensée.

- Rentrons chez nous, dit-

$\mathrm{M}^{\mathrm{me}}$ de Marelle sonna le garçon et demanda l'addition. On la lui apporta presque aussitôt. Elle essaya de la lire, mais les chiffres tournaient devant ses yeux, et elle passa le papier à Duroy : - Tenez, payez pour moi, je n'y vois plus, je suis trop grise

Et elle lui jeta en meme temps sa bourse dans les mains.

Le total montait à cent trente francs. Duroy contrồla et vérifia la note, puis donna deux billets, et reprit la monnaie, en demandant à mi-voix : Corçons?

- Ce que vous voudrez, je ne sais pas.
Il mit cing francs sur l'assiette, puis rendit la bourse Il mit cin trancs sur lassiete:

a la jeune femme, en lui disant: porte?

- Mais certainement. Je suis incapable de retrouver mon adresse.

On serra les mains des Forestier; et Duroy se trouva On serra les mains des For Il la sentait contre lui, si près, enfermée avec lui dans cette boitte noire, gu'éclairaient brusquectu pendant un instant, les becs de gaz des trottoirs. I sentait à travers sa manche, la chaleur de son épaule, et il ne trouvait rien a lui dire, absolument rien, ayant l'esprit paralysé par le désir impérieux de la saisir dans ses bras. "Si j'osais, que ferait-elle? » pensait-il. Et le souvenir de toutes les polissonneries chuchotées pendant le diner l'enhardissait, mais la peur du scandale le retenait en même temps.

Elle ne disait rien non plus, immobile, enfoncée en son coin. Il eût pensé qu'elle dormait s'il n'avait vu

briller ses yeux chaque fois qu'un rayon de lumière pénétrait dans la voiture.

"Que pensait-elle? " Il sentait bien qu'il ne fallait point parler, qu'un mot, un seul mot, rompant le silence, emporterait ses chances; mais l'audace le manquait, laudace de l action brisque et brutale Tout a coup il sentit remuer son pied. Elle avait fait un mouvement, un mouvement sec, nerveux, dimpa tience ou dappel peut-etre. Ce geste, presque insens. sur la peau, et se tournant vivement, il se jeta sur elle cherchant la bouche avec ses lèvres et la chair nue avec ses mains.

Elle jeta un cri, un petit cri, voulut se dresser, se débattre, le repousser, puis elle céda, comme si la force lui eût manqué pour résister plus longtemps. Mais la voiture setant arrêtée bientôt devant maison qu'elle habitait, Duroy, surpris, n eut point chercher des paroles passionnees pour la remercier, bénir et lui exprimer son amour reconnaissant. Cependant elle ne se levait pas, elle ne remuait point étourdie par ce qui venait de se passer. Alors craignit que le cocher n'eût des doutes, et il descend le premier pour tendre la main à la jeune femme. Elle sortit enfin du fiacre en trebuchant et san prononcer une parole. Il sonna, et, comme la pore reverrai-je?

Elle murmura si bas qu'il entendit à peine:Elle téjeuner avec moi demain. Et elle dispar dans dejeuner avec moi demain. - Et elle cispar battant qui fit un bruit de coup de canon.

Il donna cent sous au cocher et se mit à marcher devant lui, d'un pas rapide et triomphant, le coet débordant de joie.

Il en tenait une, enfin, une femme mariée! un

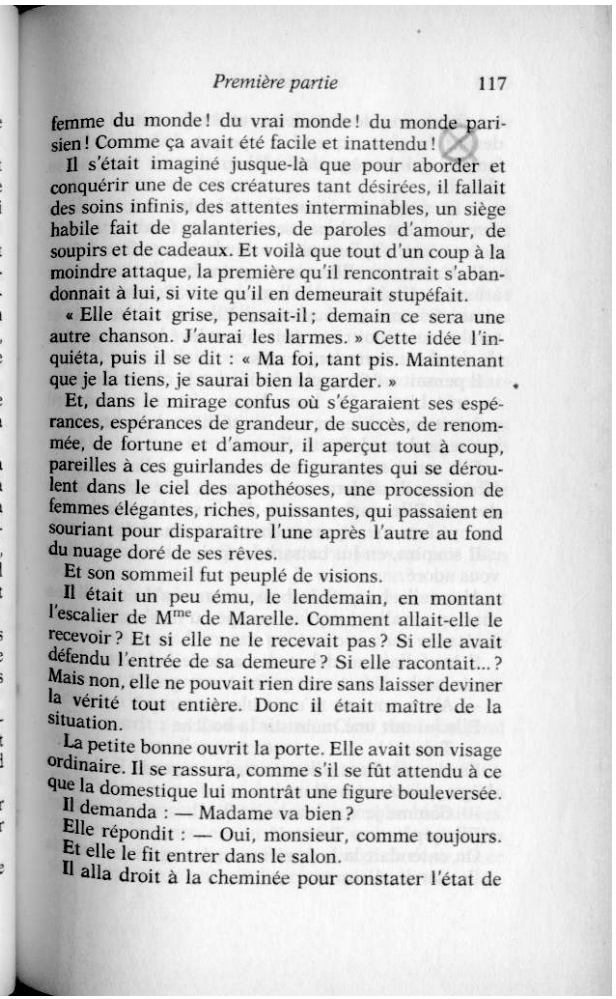

\section{Troisième passage de Bel-Ami de Maupassant :}

«Bel-Ami, à genoux à côté de Suzanne, avait baissé le front. (...) l'image de Mme de Marelle rajustant en face de la glace les petits cheveux frisés de ses tempes, toujours défaits au sortir du lit. » 
414

dessus des têtes inclinées. Vauri et Landeck, de l'Opéra, chantaient. L'encens répandait une odeur fine de benjoin, et sur l'autel le sacrifice divin s'accomplissait, l Homme-Dieu, a lappel de son prêtre descendait sur la terre pour baron Georges Du Roy.

à côté de Suzanne, avait baissé le se sentait en ce moment presque croyant, presque religieux, plein de reconnaissance pour vinité qui lavait ainsi favorisé, qui le traitait avec ces égards. Et sans savoir au juste à qui il s'adressait, il la remerciait de son succès.

Lorsque l'office fut terminé, il se redressa, et, donnant le bras à sa femme, il passa dans la sacristie. Alors commença l'interminable défilé des assistants. Georges, affolé de joie, se croyait un roi qu un peuple venait acclamer. Il serrait des mains, balbutiait des mots qui ne signifiaient rien, saluait, répondait aux compliments : "Vous êtes bien aimable.

Soudain il aperçut $\mathrm{M}^{\mathrm{me}}$ de Marelle; et le souvenir de tous les baisers qu'il lui avait donnés, qu'elle lui avai rendus, le souvenir de toutes leurs caresses, de se gentillesses, du son de sa voix, du goût de ses lèvres lui fit passer dans le sang le desir brusque de la reprendre. Elle etait jolie, elegante, avec son air gamin et ses yeux vifs. Georges pensait: a Quelle

Eile $s$ approcha, un peu timide, un peu inquiète, et Alors il sentit l'appel discret de ces doigts de femme. la douce pression qui pardonne et reprend Ft luimême il la serrait, cette petite main, comme pour dire : « Je t'aime toujours, je suis à toi! "

Leurs yeux se rencontrèrent, souriants, brillants, pleins damour. Elle murmura de sa voix gracieuse - A bientôt, monsieur.

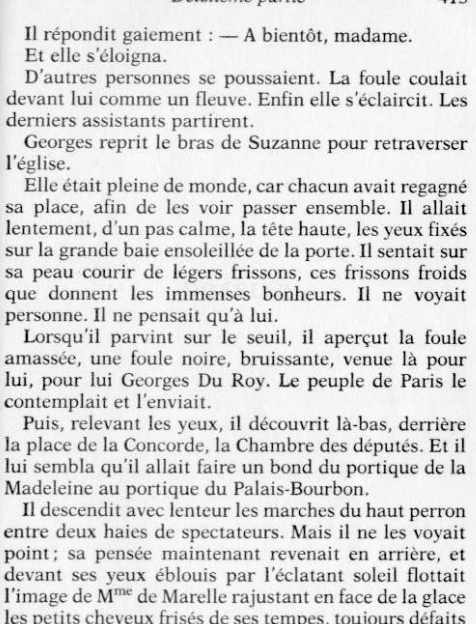

Elle était pleine de monde, car chacun avait regagné sa place, afin de les voir passer ensemble. Il allait sur la grande baie ensoleillée de la porte. Il sentait sur sa peau courir de légers frissons, ces frissons froids que donnent les immenses bonheurs. It ne voyait quersonne. Il ne pensait qu'à bon. Lorsqu'il parvint sur le seuil, il apercut la foule amassée, une foule noire, bruissante, venue là pour lui, pour lui Georges Du Roy. Le peuple de Paris le contemplait et l'enviait.

Puis, relevant les yeux, il découvrit là-bas, derrière la place de la Concorde, la Chambre des députés. Et il lui sembla qu'il allait faire un bond du portique de la Madeleine au portique du Palais-Bourbon.

Il descendit avec lenteur les marches du haut perron entre deux haies de spectateurs. Mais il ne les voyait point; sa pensée maintenant revenait en arrière, et devant ses yeux éblouis par Téclatant soleil flottait les petits cheveux friśs de ses tempes, toujou dé

\section{Conventions de transcription ICOR de l'Equipe ICAR (Université Lyon II Lumière) :}

EL(E)(S) élève(s) non identifié(s)

\section{EVT événement}

SIL silence mesuré au dixième de seconde

= enchaînement immédiat

[il a dit] chevauchement de parole

(inaud.) inaudible, nombre de syll. non identifiable

(xx) inaudible, nombre de syll. identifiable

:: allongement

$<(($ en riant $))$ transcription> élément non-verbal, paraverbal

/ \montée et chute intonative

\section{NOTES}

1. Je remercie vivement Gérard Vigner d'avoir mis à ma disposition cette étude (étude non publiée).

2. Pour des raisons pratiques, nous avons calculé à part les modalisations comprises dans les glissements discursifs.

3. Miélan est un petit village d'environ 1000 habitants en milieu rural.

4. Avec 23500 habitants, la ville d'Auch, chef-lieu du Gers, est considérée comme une zone urbaine. 
5. Dans ces quatre années est comptée l'année de stage obligatoire pour les capétiens, année durant laquelle les enseignants-étudiants reçoivent une formation pédagogique et donnent des cours en école.

6. Nous estimons que cette enseignante démarre sa carrière au regard des trois autres enseignants qui ont plus d'une dizaine d'années d'expérience.

7. Après un certain nombre d'années d'enseignement dans le secondaire, un maitre auxiliaire peut demander à passer le CAPES interne afin de titulariser sa situation.

\section{RÉSUMÉS}

Reformuler en classe de langue (FLM et/ou FLES) est un acte inhérent à toute séquence de cours afin de faire comprendre du lexique. Cette opération linguistique prend forme dans l'interaction didactique et permet notamment d'expliquer du lexique difficile à appréhender pour les apprenants. Ce critère de difficulté dans l'apprentissage défini par Bogaards (1994) nous renseigne sur la nature sémantique du lexique, sachant que toutes les lexies et expressions lexicales ne sont pas égales les unes aux autres. Nous croiserons cette perspective sémantique avec une analyse interactionnelle du discours des enseignants en vue de montrer comment ces derniers jalonnent leurs discours de marques modalisatrices (Greimas, 1976).

Rephrasing in classrooms of French as a Native Language or French as a Foreign and Second Language is an inherent act in any classroom. This linguistic operation takes place in the didactic interaction and can explain some difficult vocabulary to learners. This sort of difficulty in training defined by Bogaards (1994) informs us about the semantic nature of the vocabulary. We know that the lexical items are not equal the ones to the others. Furthermore, we aim to cross a semantic perspective with an interactional analysis of teachers'language in order to show modalizing in their discourses (Greimas, 1976).

\section{INDEX}

Keywords : rephrasing, interaction, teachers' discourse, lexicon categorization, modalizing Mots-clés : reformulation, interaction, discours enseignant, catégorisation lexicale, modalisation

\section{AUTEUR}

\section{JULIE RANÇON}

Université de Poitiers

Laboratoire FoReLL - EA 3816 